The Astrophysical Journal, 197:443-455, 1975 April 15

(C) 1975. The American Astronomical Society. All rights reserved. Printed in U.S.A.

\title{
THE X-RAY, OPTICAL, AND RADIO BEHAVIOR OF SCORPIUS X-1: THE 1971 COORDINATED OBSERVATIONS
}

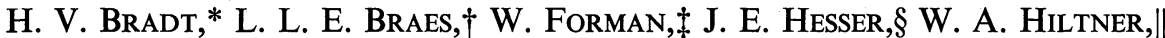 \\ R. Huellming,\# E. KellogG, $\ddagger$ W. E. Kunkel, $\S$ G. K. Miley,† G. Moore, ${ }^{*}$ \\ J. W. Pel, $\dagger$ J. Thomas, $\|$ P. VAnden Bout, ** C. WAde,\# And B. WARneR** \\ Received 1974 August 12
}

\begin{abstract}
Scorpius X-1 has been monitored at radio, optical, and X-ray wavelengths for 21 days during the period 1971 February 23 through 1971 April 2. The Uhuru satellite was used for the X-ray observations. The X-ray intensity was found to fluctuate rapidly (time constants $<6$ minutes) by a factor of about 2 during periods when the object was in a bright and flaring optical state. The X-ray flux was constant to within $\sim 5$ percent during the faint and relatively quiescent optical periods. No correlation between the X-ray/optical activity and radio flaring was noted.
\end{abstract}

Subject heading: X-ray sources

\section{INTRODUCTION}

Scorpius $\mathrm{X}-1$ is highly variable and apparently aperiodic in the three wavelength regimes (radio, optical, and X-ray) at which it has been extensively studied. Studies of the variability and spectra at the several wavelengths have not led to a well established model for the emission process or the energy source. However, a recent theoretical study by Ramaty, Cheng, and Tsuruta (1974) does take into account many of the presently known characteristics of Sco $\mathrm{X}-1$ at X-ray and radio wavelengths. Simultaneous $\mathrm{X}$-ray, radio, and optical observations should place further constraints upon possible models.

At optical wavelengths, Sco X-1 ranges in brightness from $B \approx 13.6$ to $B \approx 12.2$ on time scales of hours to days (Hiltner and Mook 1967a, b; Mook 1967). In the bright state it is very active, frequently flaring $\sim 0.2$ mag with 1-10 minute time constants. Flickering at $0.02 \mathrm{mag}$ on a time scale of $\leqslant 1$ minute is frequently present (Hiltner and Mook 1967a, b; Westphal, Sandage, and Kristian 1968). At X-ray wavelengths, Sco $\mathrm{X}-1$ does not exhibit the extreme variability on the $\leqslant 10 \mathrm{~s}$ time scale that is characteristic of the very active X-ray sources Cir X-1 and Cyg X-1 (Giacconi

* Center for Space Research, MIT, Cambridge, Massachusetts; work supported in part by the Alfred P. Sloan Foundation, MIT grant 287.

$\dagger$ Leiden Observatory, The Netherlands.

¥Center for Astrophysics, Smithsonian Astrophysical Observatory/Harvard College Observatory, Cambridge, Massachusetts; work supported in part by NASA grant NGR 09-015-211.

\& Cerro Tololo Inter-American Observatory, operated by the Association of Universities for Research in Astronomy, Inc., under contract with the National Science Foundation.

II University of Michigan, Ann Arbor, Michigan; work supported in part by NASA grant NGR 23-005-464.

\# National Radio Astronomy Observatory, Green Bank, West Virginia, operated by Associated Universities, Inc., under contract with the National Science Foundation.

** University of Texas, Austin; work supported in part by NASA grant NGR 44-012-209. et al. 1974). However, it does exhibit $\sim 50$ percent changes of intensity in $\sim 2$ minutes (Evans et al. 1970) and flaring by a factor of 4 at energies $\geq 20 \mathrm{keV}$ (Lewin, Clark, and Smith 1968). Extended OSO 3, OSO 7, and Uhuru satellite observations show long periods (1-5 days) when Sco X-1 is in a quiescent $\mathrm{X}$-ray state, and others when it is very active and flaring by factors of 2 or more (Pelling 1973; Canizares et al. 1973; Forman et al. 1971). At radio wavelengths, Sco X-1 exhibits major flaring with time constants of $\sim 1$ hour about 15 percent of the time. At $11 \mathrm{~cm}$, the unusually strong flare of 1971 February 26 exceeded $260 \mathrm{mJy}\left(1\right.$ millijansky $\left.=10^{-29} \mathrm{Wm}^{-2} \mathrm{~Hz}^{-1}\right)$. The source is quiescent ( $<3 \mathrm{mJy}$ at $11 \mathrm{~cm}$ ) about 20 percent of the time (Hjellming and Wade 1971; Wade and Hjellming 1971). This activity pertains to an unresolved point source at the location of the optical star. Two weak and apparently nonvariable satellite radio sources which lie 1'.2 on either side of the star, may be related to Sco X-1 (Wade and Hjellming 1971; Braes and Miley 1971a).

Previously published simultaneous observations have been limited, mostly due to the few satellite and rocket opportunities for X-ray observations. Hiltner (1973) has reviewed most of this work. Sounding rocket observations from eight flights yielded the first evidence for a positive correlation between the X-ray and optical flux (Burginyon et al. 1970). Another rocket flight yielded evidence for a simultaneous $\mathrm{X}$ ray/optical 20-s periodicity which lasted about 2 minutes and consisted of 0.8 and 0.6 percent of the total X-ray and optical intensities, respectively (Kestenbaum et al. 1971). Balloon observations by Matsuoka et al. (1972) showed a positive correlation between the X-ray (20-30 keV) flux and the optical $B$-magnitude flux during an $\sim 8$-minute optical flare.

Vela 5 satellite observations correlated with optical observations showed a major increase in optical brightness ( $B=13.1$ to $B=12.7$ ) over a period of 1 hour which was followed $\sim 1.5$ hours later by an increase in X-ray intensity by a factor of $\geq 2.5$ (Evans 
et al. 1970). Just prior to this, during the early part of the optical increase, the X-ray intensity steadily decreased by about 30 percent in 1 hour. The OSO 3 satellite $(7.7-12.5 \mathrm{keV})$ yielded four coincidences between 10 and 20 minute optical flares $(\sim 0.1 \mathrm{mag})$ observed at Mount Palomar and an enhanced X-ray flux (Pelling 1973). The same work showed that, during the observations, the X-ray intensity reached its maximum values when the optical intensity was high. Extended simultaneous X-ray (OSO 7) and radio (NRAO) observations have been reported by Canizares et al. (1973). They show no apparent correlation between the radio and $\mathrm{X}$-ray activity.

Three major collaborative efforts to obtain simultaneous observations of Sco X-1 were organized by various groups in 1970,1971, and 1972, to take advantage of extended X-ray observations by the Vela 5 , Uhuru, and OSO 7 satellites. The present paper is a summary of the results from the 1971 Uhuru observations. Portions of the data have been presented in the review by Hiltner (1973).

\section{OBSERVATIONS}

The optical data reported here were obtained at four observatories: Cerro Tololo, McDonald, Leiden Southern Station, and Kitt Peak. For the most part, the observations consisted of straightforward monitoring of the $B$ magnitude with a time resolution of $\sim 30 \mathrm{~s}$. The Leiden data were obtained in the fivecolor $V B L U W$ system, and the McDonald data were obtained with an Amperex 56DVP blue-sensitive photomultiplier without filters. The latter data were matched to Kitt Peak magnitudes during periods of simultaneous observations. In the former case, we assumed that the five-color $B$ magnitude varies proportionately with the three-color $(U B V) B$ magnitude. In general, $B$ magnitudes for the individual $\sim 30 \mathrm{~s}$ integrations are precise to $0.02 \mathrm{mag}$ or better. The observations are summarized in table 1.

Radio data were obtained from the Westerbork synthesis telescope at $1415 \mathrm{MHz}(21.2 \mathrm{~cm})$, and the NRAO interferometer at 2695 and $8085 \mathrm{MHz}(11.1$ and $3.7 \mathrm{~cm}$ ). Most of these data have been published (Braes and Miley 1971b; Wade and Hjellming 1971). The Westerbork intensities were derived from integrations of $\sim 20$ minutes during active periods and $\sim 2$ hours during quiet periods. The uncertainties in these measurements are $\sim 6$ and $\sim 2 \mathrm{mJy}$ for the shorter and longer integrations, respectively. The NRAO data intensities typically are derived from 25minute integrations with uncertainties of $\sim 5 \mathrm{mJy}$. Herein, we plot the 11.1-cm data, rather than the 3.7 $\mathrm{cm}$, because the variability is more pronounced.

The X-ray data were obtained from the Uhuru satellite. They consist of discrete measurements $(2.4-6.9 \mathrm{keV})$ of the X-ray intensity once every $\sim 6$ minutes by two banks of counters on opposite sides of the spinning spacecraft. The detection systems have been described by Giacconi et al. (1971) and Jagoda et al. (1972). The X-ray collimators on the two counter banks limit the durations of the alternating measure-
TABLE 1

Optical ObServations

\begin{tabular}{|c|c|c|c|}
\hline & Date (1971) & Time $(h, \mathrm{UT})$ & Observatory \\
\hline Mar. & 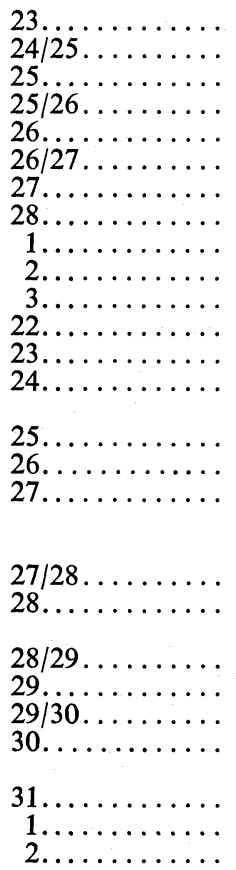 & $\begin{array}{r}6.9-9.3 \\
23.6-3.1 \\
7.2-9.4 \\
23.9-2.9 \\
7.1-9.3 \\
23.4-3.0 \\
8.6-9.6 \\
6.6-8.7 \\
6.1-8.8 \\
6.2-8.9 \\
6.0-9.4 \\
6.3-9.7 \\
5.3-9.3 \\
5.1-9.6 \\
9.9-11.1 \\
4.8-9.6 \\
5.2-9.2 \\
4.7-9.6 \\
8.6-10.0 \\
8.8-13.0 \\
23.4-2.6 \\
4.5-9.5 \\
9.1-10.5 \\
23.4-3.2 \\
8.4-12.3 \\
21.6-3.3 \\
5.1-7.6 \\
8.3-12.1 \\
5.0-9.6 \\
4.3-9.4 \\
4.8-9.7\end{array}$ & $\begin{array}{l}\text { CTIO* } \\
\text { LSS } \dagger \\
\text { CTIO } \\
\text { LSS } \\
\text { CTIO } \\
\text { LSS } \\
\text { CTIO } \\
\text { CTIO } \\
\text { CTIO } \\
\text { CTIO } \\
\text { CTIO } \\
\text { CTIO } \\
\text { CTIO } \\
\text { CTIO } \\
\text { MO } \\
\text { CTIO } \\
\text { CTIO } \\
\text { CTIO } \\
\text { MO } \\
\text { KPNO } \\
\text { LSS } \\
\text { CTIO } \\
\text { MO } \\
\text { LSS } \\
\text { KPNO } \\
\text { LSS } \\
\text { CTIO } \\
\text { KPNO } \\
\text { CTIO } \\
\text { CTIO } \\
\text { CTIO }\end{array}$ \\
\hline
\end{tabular}

* Cerro Tololo Inter-American Observatory; Observers: H. B. and J. H.; Telescopes: 36-inch $(91 \mathrm{~cm}$ ) (February 23, $25,26)$; 16-inch (41 cm) (February 27-March 3); 24-inch $(61 \mathrm{~cm})$ (March 22-April 2).

$\dagger$ Leiden Southern Station: observer: J. P.; telescope: 36-inch $(91 \mathrm{~cm})$.

$\ddagger$ McDonald Observatory; observers: B. W. and P. V.; telescope: 82 -inch $(2 \mathrm{~m})$.

$\S$ Kitt Peak National Observatory; observer: W. H.; telescope: 36 -inch $(91 \mathrm{~cm})$.

ments to $\sim 2$ and $\sim 20 \mathrm{~s}$, respectively. The satellite aspect solution was used to carry out a least-squares fit to the data of each transit, because the effective areas of the detectors depend upon the position of Sco X-1 in the fields of view of the collimators. The source intensity was assumed to be constant during the transit, and the minimum $\chi^{2}$ determined the intensity reported herein. The number of X-ray measurements was limited by Earth occultations and by the requirement that the satellite be in direct contact with a ground station. Approximately 550 measurements of the intensity were obtained with combined errors (statistics and aspect) which range typically from $\sim 2$ to $\sim 4$ percent, though they are occasionally larger when Sco X-1 transits near the edge of the field of view. The two banks of counters were calibrated relative to each other, to within $\sim 3$ percent on the basis of preflight data and $\sim 10$-day averages of the quiescent Sco X-1 data. Occasionally, a small residual offset can be noted in the data presented here. We will not present here an analysis of the variability 
during single transits. Thus the results will pertain only to X-ray variability with time constants $\tau \geqslant$ 6 minutes.

\section{RESULTS}

The results of the simultaneous observations are presented in figures 1-9. In each case, the plots are linear in intensity, though the zero intensity is offscale for the X-ray and optical data. Figure 1 shows the entire data for the 21 days of observations, a 9-day run in 1971 February-March and a 12-day run in 1971 March-April. Figures 2-4 show the same data, with an expanded horizontal scale such that most of the features $(\tau \geqslant 10$ minutes) in the data can be resolved. In figures $5-8$, we present portions of the data with a further expansion of the time axis to permit an examination of simultaneous features on the $\sim 30$-s time scale intrinsic to the present analysis. Most of the coincident optical/X-ray data in the March-April run are so presented. Finally, in figure 9, we summarize the data with intensity distributions (radio, optical, and X-ray) and an X-ray/optical scatter plot.

A perusal of the figures shows that the behavior of Sco X-1 in the three wavelength bands is very complex. In figure 1, we note periods of great variability (more than a factor of 2) and increased average brightness in the X-ray intensity (e.g., March 27), interspersed with periods of less intense and steadier emission (e.g., February 26). The active X-ray periods appear to coincide directly with the active (bright and possibly flaring) optical periods. This correlation is probably real because of the lack of clear counterexamples and the near coincidences of the turnon of March 26 and the turnoff of March 28/29. The spectacular radio flare (peak amplitude $>260 \mathrm{mJy}$ ) of February 26 is seen to occur in a period when X-ray and optical signals are quiescent, though exactly coincident observations were not obtained. Two other periods of radio activity (March 25 and 30/31) also occur during quiet X-ray/optical periods.

The variation of the optical intensity in figure $1 b$ has the appearance of an $\sim 5$-day period with a minimum of $\sim 1.5$ days. This is reminiscent of the 3.9-day period recently suggested by Lyutyj and Efremov (1974). However, our data do not easily match such a period. (The reported period and phase predict minima at days 21.4, 25.4, 29.3, and 2.2 on fig. 1b.) We note that the data of Mook (1967) are inconsistent with a 4-5 day periodicity with the $\sim 1.5$-day minimum we observe. He observed Sco $\mathrm{X}-1$ to remain in a bright state for $\sim 7$ nights and again for $\sim 8$ nights.

In figures 2-4, we note the following: at $\sim 7^{\mathrm{h}}$ UT February 23, a coincident X-ray/optical brightening (the arrow marks the time of the highest X-ray point); at $0^{\mathrm{h}}-3^{\mathrm{h}}$ February 25 , significant variability on time scales of $\sim 1$ hour in the faint optical state; at $0^{\mathrm{h}}-9^{\mathrm{h}}$ February 25, the constancy of the quiescent X-ray state to within $\sim 5$ percent; at $7^{\mathrm{h}}$ March 22 and $6^{\mathrm{h}}$ March 24, an apparent coincident X-ray/optical decrease in intensity; at $5^{\mathrm{h}}-9^{\mathrm{h}}$ March 25 and on March $29 / 30$, a significant $(\sim 30$ percent $)$ decrease in the optical flux while the X-ray flux holds constant; at $8^{\mathrm{h}}-11^{\mathrm{h}}$ March 26, the "turnon" or change of state of the optical $\sim 1 \frac{1}{2}$ hours prior to an X-ray "turnon"; and at $\sim 0^{\mathrm{h}}$ March 28/29, an optical rise and fall which may be part of the major optical turnoff (see March 28-30 in fig. 1b). The rise and fall take place when the $\mathrm{X}$-ray flux is quiescent $\sim 5$ hours after a return from the active state.

In figures 5-8, features mentioned above can be seen in more detail; e.g., on March 25, the slow optical decline during a constant X-ray state; on March 26, the optical change of state followed by the X-ray increase; and on March 28/29, the optical rise and fall. Also we note apparent coincidences between $\mathrm{X}$-ray and optical features (see arrows) on March $22,23,24,27$, and 28 . The times of a few other X-ray points which are near optical features are marked with vertical lines to guide the eye. Although these data appear to be consistent with the existence of coincident X-ray and optical $\sim 15$-minute flares, the relatively long time interval ( $\sim 6$-minute) between the brief X-ray sightings $(2-20 \mathrm{~s})$ prevents us from drawing such a conclusion. Also, we note clear instances where the X-ray intensities undergo marked variations which do not appear to be reflected in a one-to-one variation in the optical flux, i.e., the decreases which occur at 0850-0900 March 27 and 0840-0850 March 28.

In figure 9, we note that the optical distribution of intensities is bimodal, similar to the 1967 distribution reported previously (Hiltner and Mook 1970). (Note that we plot frequency against a linear intensity scale, rather than $B$ magnitude, to permit a comparison to the X-ray and radio histograms. This serves to enhance the faint peak.) The X-ray distribution (fig. $9 c$ ) does not exhibit this effect but has a high-intensity tail. This is consistent with the observation of Canizares et al. (1973) that Sco X-1 appears to enter its flaring X-ray state directly from a low quiescent state. The radio distributions (fig. 9a) are more characteristic of the X-ray distribution. Finally, the X-ray/ optical scatter plot (fig. 9d) shows the great X-ray variability when the optical is bright. Note that there is no case of a high X-ray flux when the optical flux is faint.

\section{CONCLUSIONS}

One can draw several conclusions about these data:

1) The X-ray intensity at $2.4-6.9 \mathrm{keV}$ is often high and variable by more than a factor of 2 with time constants $\leqslant 6$ minutes. This activity can last for several days at a time. During intervening periods, also of several days duration, the X-ray intensity is low, and constant to within $\sim 5$ percent. This quiescent behavior may be unique among stellar X-ray sources.

2) The distribution of optical intensities during these observations is bimodal. Flares of 0.1-0.2 mag are rather frequently observed in the bright state and are never observed in the faint state. In the faint state, the optical intensity can slowly change by $\sim 30$ percent in several hours, while the X-ray intensity remains low and constant. 
3) The active X-ray state and the bright optical state are apparently always coincident. A turnon, or "change of state," was observed wherein an increase of optical intensity by a factor of $\sim 2$ was followed after $\sim 1 \frac{1}{2}$ hours by a similar increase in the X-ray intensity. This is similar to the Vela 5 result mentioned earlier. In another instance, the optical appeared to "turn on," but after $\sim 1 \frac{1}{2}$ hours reversed itself, all with no apparent effect upon the X-ray intensity. However, it does follow an extended period of X-ray and optical activity, and may represent part of the "turnoff" of the source.

4) There are several instances of a high X-ray intensity coincident with the peak of an optical flare. However, the relatively long duration $(\geqslant 6$ minutes) between the brief X-ray samples (2-20 s), and the generally chaotic behavior of the signals during active periods, prevent us from drawing a firm conclusion about the reality of such coincidences.
5) Radio flaring occurred only during quiet X-ray/ optical periods. No apparent correlation or systematic delay is evident.

We express our gratitude to the entire staffs of the several observatories and institutions at which this work was performed. We particularly thank $\mathrm{R}$. Giacconi, H. Gursky, and H. Tananbaum for helping to make available the X-ray data used herein, and for their role in the conception and support of the Uhuru satellite. We also thank the staff at American Science and Engineering, Inc., for their support of the Uhuru mission. We are grateful to R. Shafer of MIT for his thoughtful assistance in the preparation of the data for publication. One of us (H. B.) gratefully acknowledges the support he received from the Cerro Tololo Inter-American Observatory during his sabbatical leave at that institution.

\section{REFERENCES}

Braes, L. L. E., and Miley, G. K. 1971a, Astr. and Ap., 14,

160. $1971 b$, Bamberger Veröff, No. 100, 9, 173.

Hiltner, W. A., and Mook, D. E. 1967b, Ap. J., 150, 851.

- 1970, Ann. Rev. Astr. and Ap., 8, 139.

Burginyon, G. A., Grader, R. J., Hill, R. W., Price, R. E., Rodrigues, R., Seward, F. D., Swift, C. D., Hiltner, W. A., and Mannery, E. J. 1970, Ap. J., 161, 987.

Canizares, C. R., Clark, G. W., Lewin, W. H. G., Schnopper, H. W., and Sprott, G. F. 1973, Ap. J. (Letters), 179, L1.

Evans, W. D., Belian, R. D., Conner, J. P., Strong, I. B., Hiltner, W. A., and Kunkel, W. E. 1970, Ap.J. (Letters), 162, L115.

Forman, W., Kellogg, E., Gursky, H., Tananbaum, H., Giacconi, R., Bradt, H. V., Moore, G., Kunkel, W. E., Hiltner, W. A., Thomas, J., Warner, B., and Vanden Bout, P. 1971, Bull. AAS, 3, 457.

Giacconi, R., Kellogg, E., Gorenstein, P., Gursky, H., and Tananbaum, H. 1971, Ap. J. (Letters), 165, L27.

Giacconi, R., Murray, S., Gursky, H., Kellogg, E., Schreier, E., Matilsky, T., Koch, D., and Tananbaum, H. 1974, Ap. J. Suppl., No. 237, $27,37$.

Hiltner, W. A. 1973, in $X$ - and Gamma-ray Astronomy, IAU Symp. No. 55, ed. H. Bradt and R. Giacconi (Dordrecht and Boston: Reidel), p. 74.

Hiltner, W. A., and Mook, D. E. 1967a, Ap. J. (Letters), 150, L23.

Hjellming, R. M., and Wade, C. M. 1971, Ap. J. (Letters), 164, L1.

Jagoda, N., Austin, G., Mickiewicz, S., and Goddard, R. 1972, IEEE, Trans. Nucl. Sci., NS-19, No. 1, p. 579.

Kestenbaum, H., Angel, J. R. P., Novick, R., and Cocke, W. J. 1971, Ap. J. (Letters), 169, L49.

Lewin, W. H. G., Clark, G. W., and Smith, W. B. 1968, Ap. J. (Letters), 152, L55.

Lyutyj, V. M., and Efremov, Yu. N. 1974, IAU Circ., No. 2675 (also see IAU Circ., No. 2679, and Lyutyj, V. M., Sunyaev, R. A., and Shakura, N. I. 1974, Astr. Zh., 51, No. 5, p. 905).

Matsuoka, M., Fujii, M., Miyamoto, S., Nishimura, J., Oda, M., Ogawara, Y., Ohta, S., Hayakawa, S., Kasahara, I., Makino, F., Tanaka, Y., Agrawal, P. C., Sreekantan, B. V., Hatanaka, Y., and Sreedhar Rao, S. 1972, Nature Phys. Sci., 236, 53.

Mook, D. E. 1967, Ap. J. (Letters), 150, L25.

Pelling, R. M. 1973, Ap. J., 185, 327.

Ramaty, R., Cheng, C. C., and Tsuruta, S. 1974, Ap. J., 187, 61.

Wade, C. M., and Hjellming, R. M. 1971, Ap. J., 170, 523.

Westphal, J. A., Sandage, A., and Kristian, J. 1968, Ap. J., 154, 139.

HALE V. BRADT: Room 37-581, Massachusetts Institute of Technology, Cambridge, MA 02139

L. L. E. Braes, G. K. Miley, and Jan Willem Pel: Sterrewacht te Leiden, Leiden, The Netherlands

William Forman and Edwin Kellogg: Center for Astrophysics, 60 Garden Street, Cambridge, MA 02138

JAMEs E. HesSer and William E. KunKel: Observatorio Interamericano de Cerro Tololo, Casilla 63-D, La Serena, Chile

W. A. Hiltner and John Thomas: Department of Astronomy, University of Michigan, Ann Arbor, MI 48104

R. M. HJellming and Campbell M. Wade, JR.: National Radio Astronomy Observatory, Edgemont Road, Charlottesville, VA 22901

Gregory Moore: 316 Dana Hall, Harvard University Law School, Cambridge, MA 02138

Paul Vanden Bout: Astronomy Department, PMA 15.212, University of Texas, Austin, TX 78712

BRIAN WARNER: University of Capetown, Capetown, South Africa 

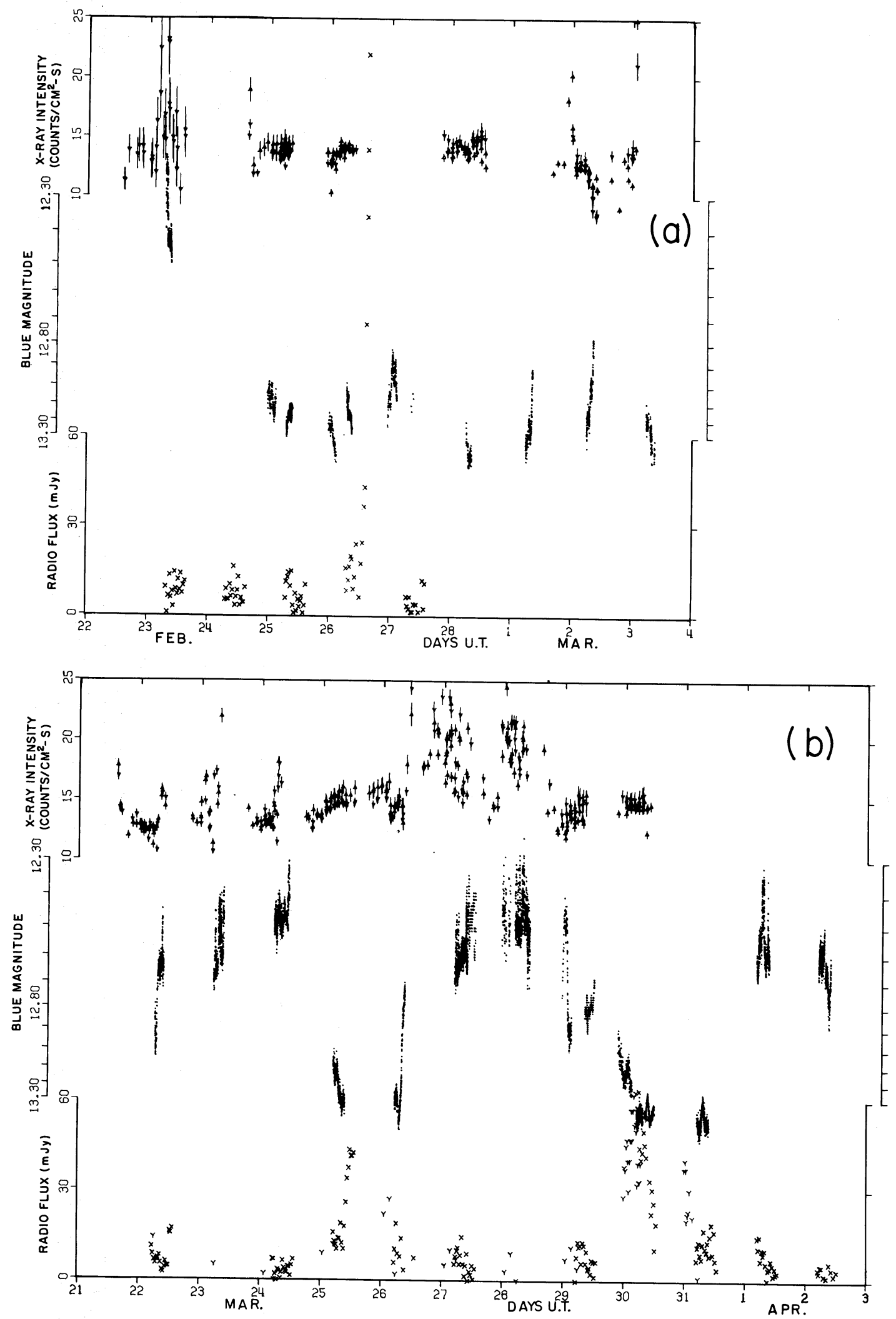

FIG. 1.-The intensities obtained during the two observing runs. The tick marks on the abscissa represent $0^{\mathrm{h}}$ UT of the day indicated. The ordinates are all linear with intensity; the optical zero $(B=\infty)$ lies on the radio scale at $\sim 6 \mathrm{mJy}$. The X-ray data $(2.4-6.9 \mathrm{keV})$ are represented by the symbols, "point-up" and "point-down," which indicate the $0.5 \times 5^{\circ}$ and $5^{\circ} \times 5^{\circ}$ Uhuru collimators, respectively. The large error bars on Feb. 23 arise because Sco X-1 was near the edge of the field of view. Thuru colintensities are shown as $X \mathrm{~s}$ for the NRAO on Feb. 23 arise because Sco X-1 was near the edge of the field of view. The radio was followed to $260 \mathrm{mJy}$, or $33 \%$ higher than shown here and $Y \mathrm{~s}$ for the Westerbork data at $21.2 \mathrm{~cm}$. The Feb. 26 radio flare 


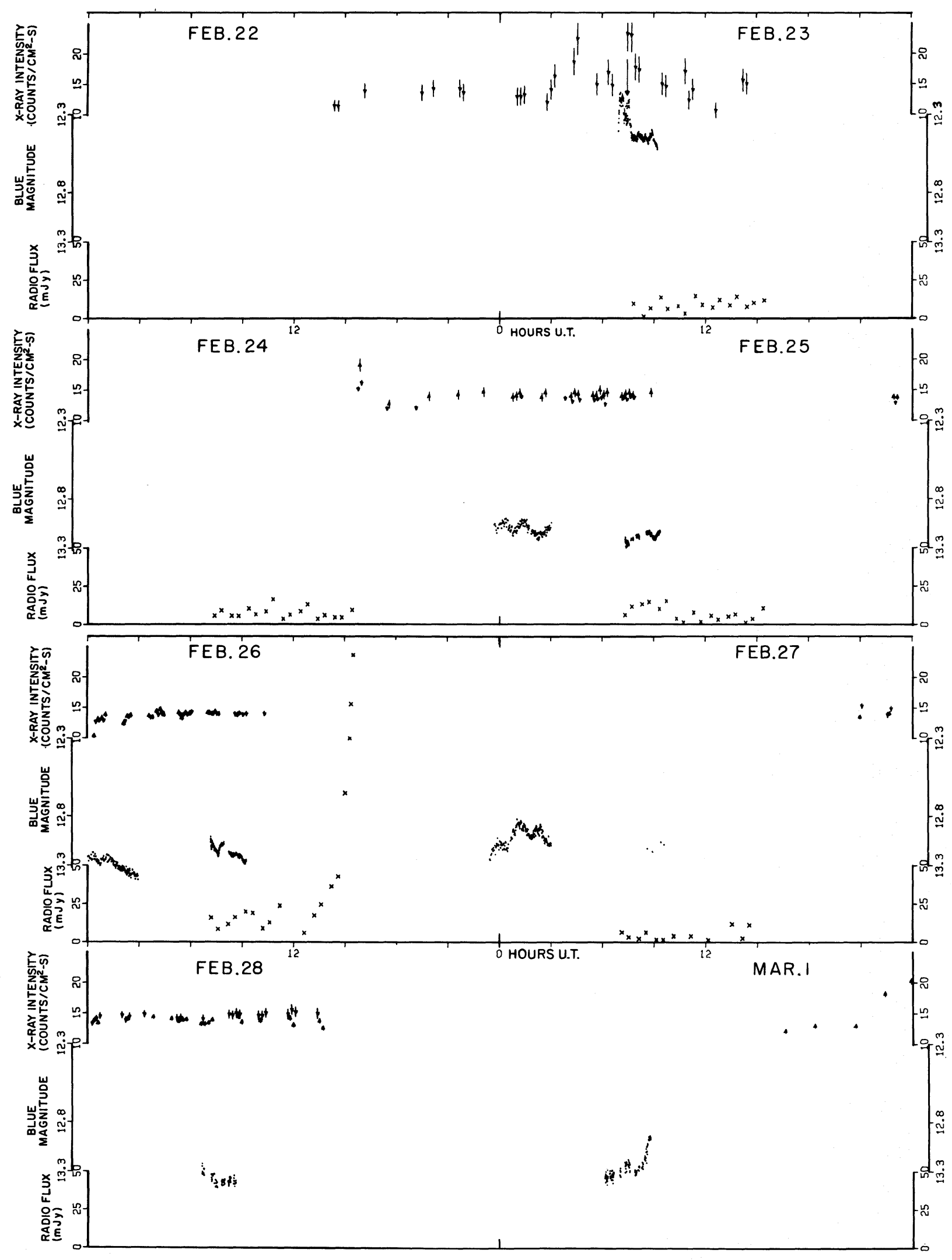

FIG. 2.-The first 8 days of the program, 1971 Feb. 22-Mar. 1. See caption to fig. 1. In figs. 2-4, the zero of optical intensity lies at $\sim-5 \mathrm{mJy}$ on the radio scale. The arrow (Feb. 23) marks the time of an X-ray point which is apparently coincident with an optical feature. 


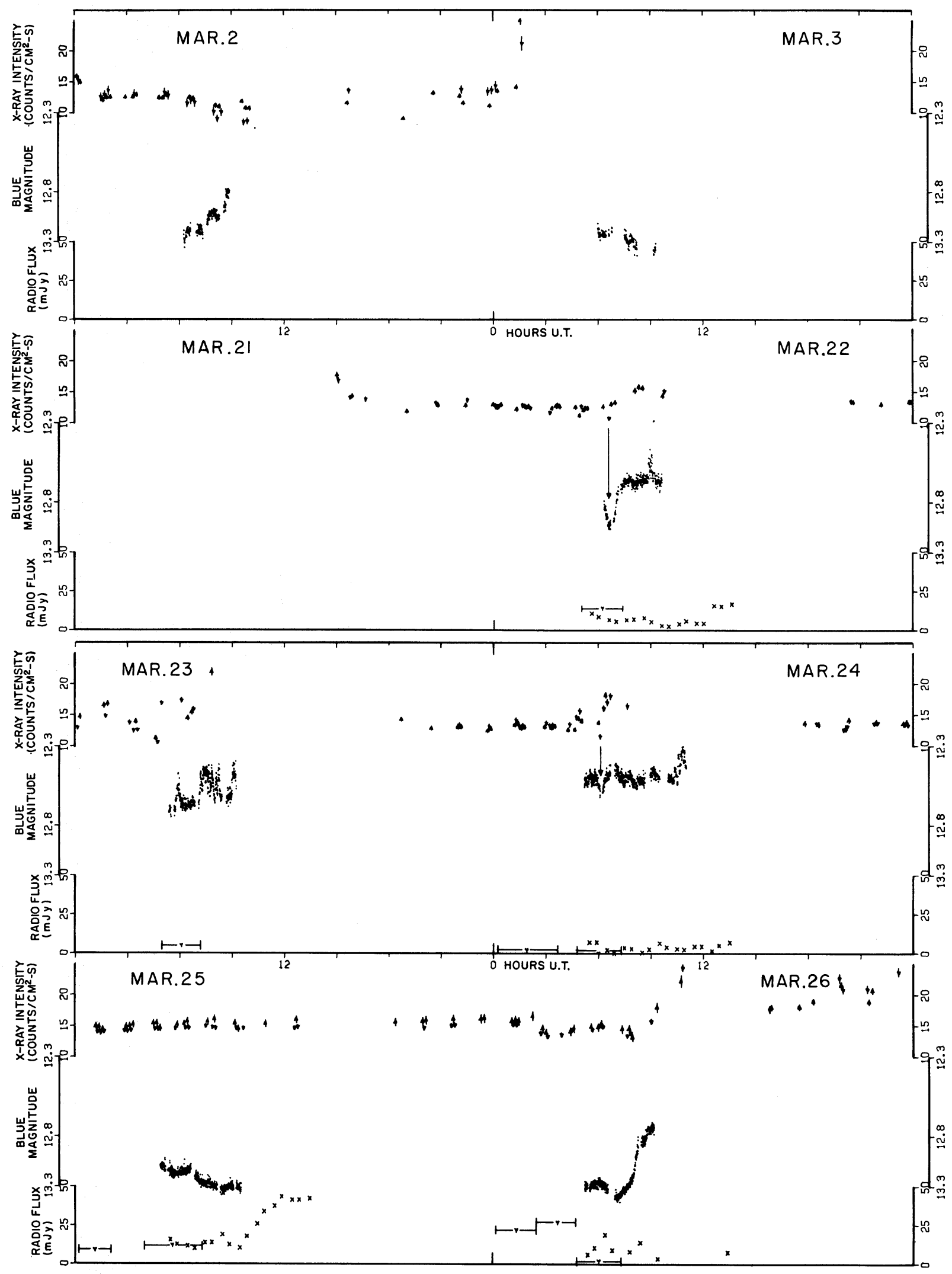

FIG. 3. - The final two days of the first run, 1971 Mar. 2-3, and the beginning of the second run, 1971 Mar. 21-26. See captions to figs. 1 and 2. The horizontal bar on the Westerbork data points (e.g., Mar. 22) indicates the duration of the integration period. 


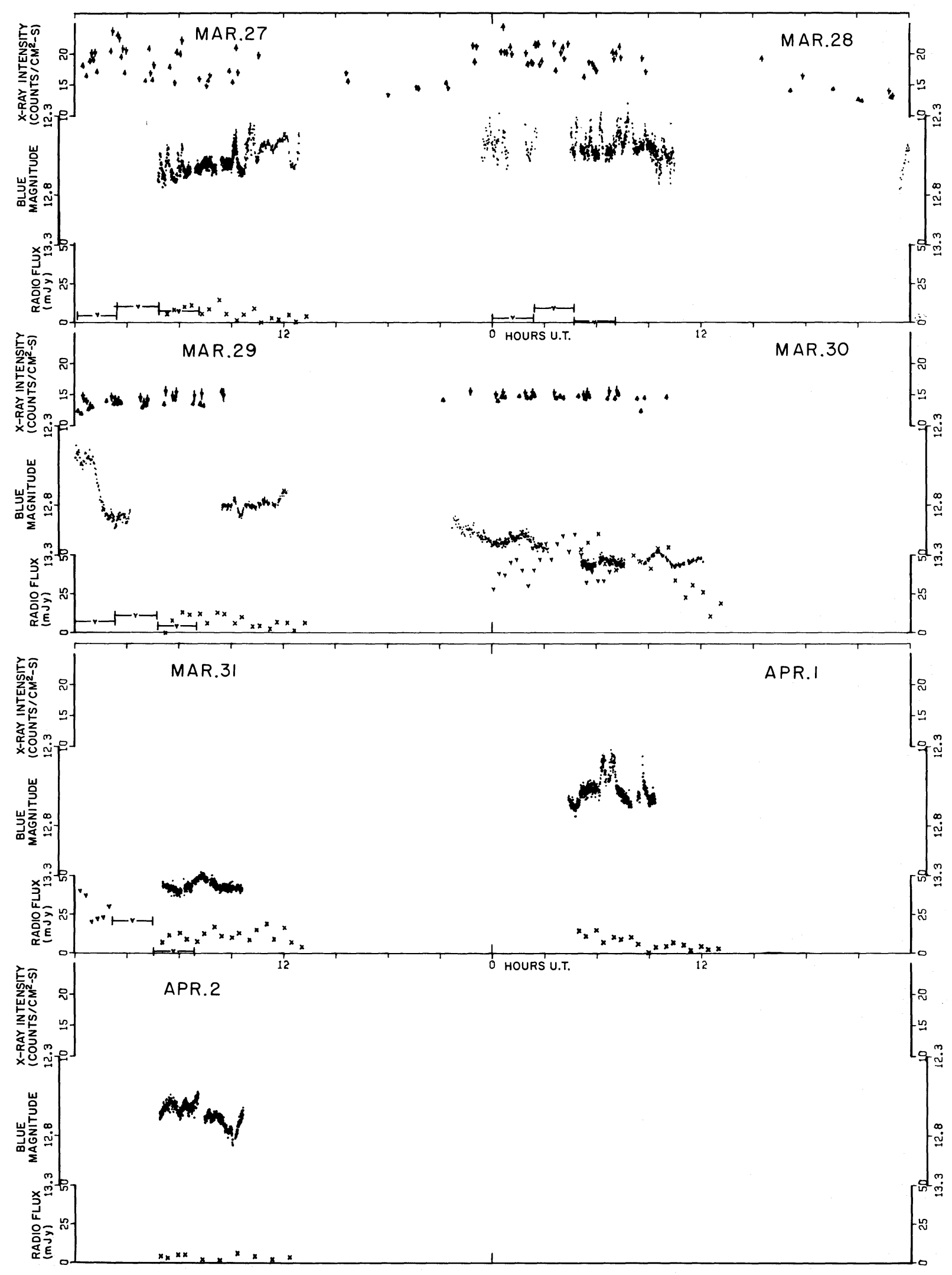

FIG. 4.-The final 7 days, 1971 Mar. 27-Apr. 2

450 

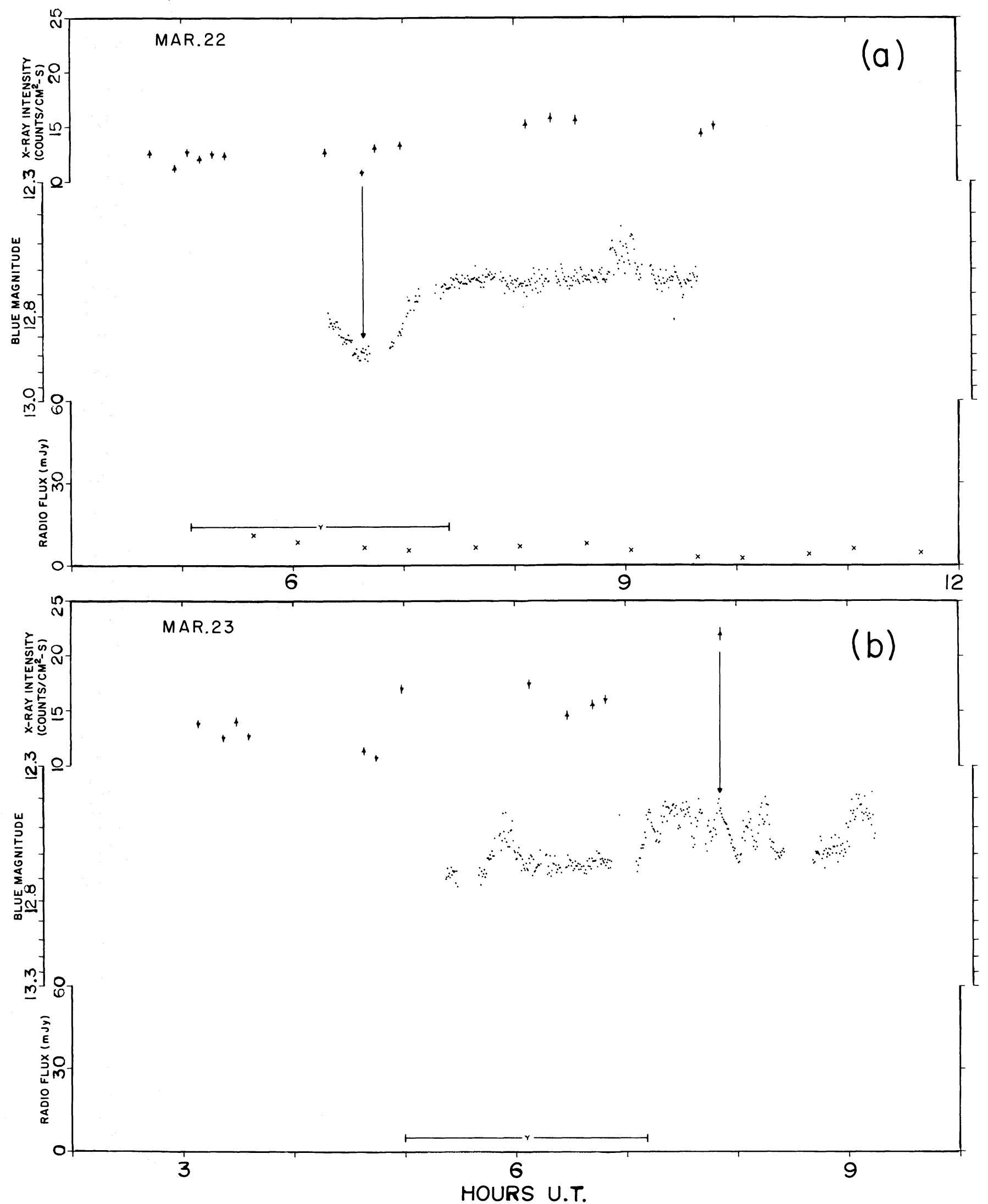

Fig. 5.-Expanded segments of the data on Mar. 22 and Mar. 23. The zero of optical intensity is at $\sim 6 \mathrm{mJy}$. The horizontal bars indicate the integration periods for the Westerbork data. The vertical arrows indicate the times of X-ray points which are in apparent, but possibly accidental, coincidence with optical features. The vertical lines under other points (e.g., fig. 7) are for reference only. 

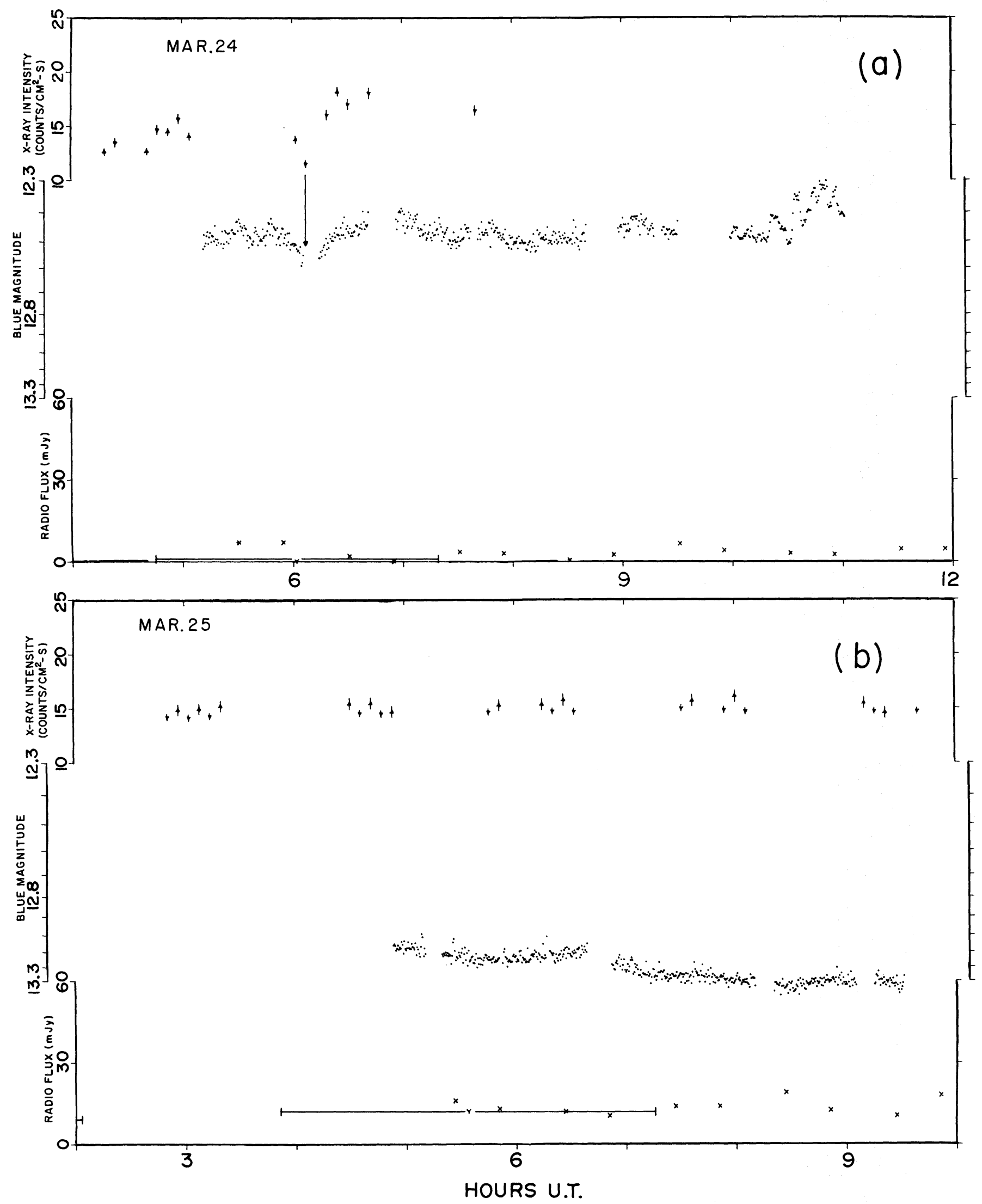

Fig. 6.-Expanded segments of the data, Mar. 24 and Mar. 25. See legend to fig. 5.

452 

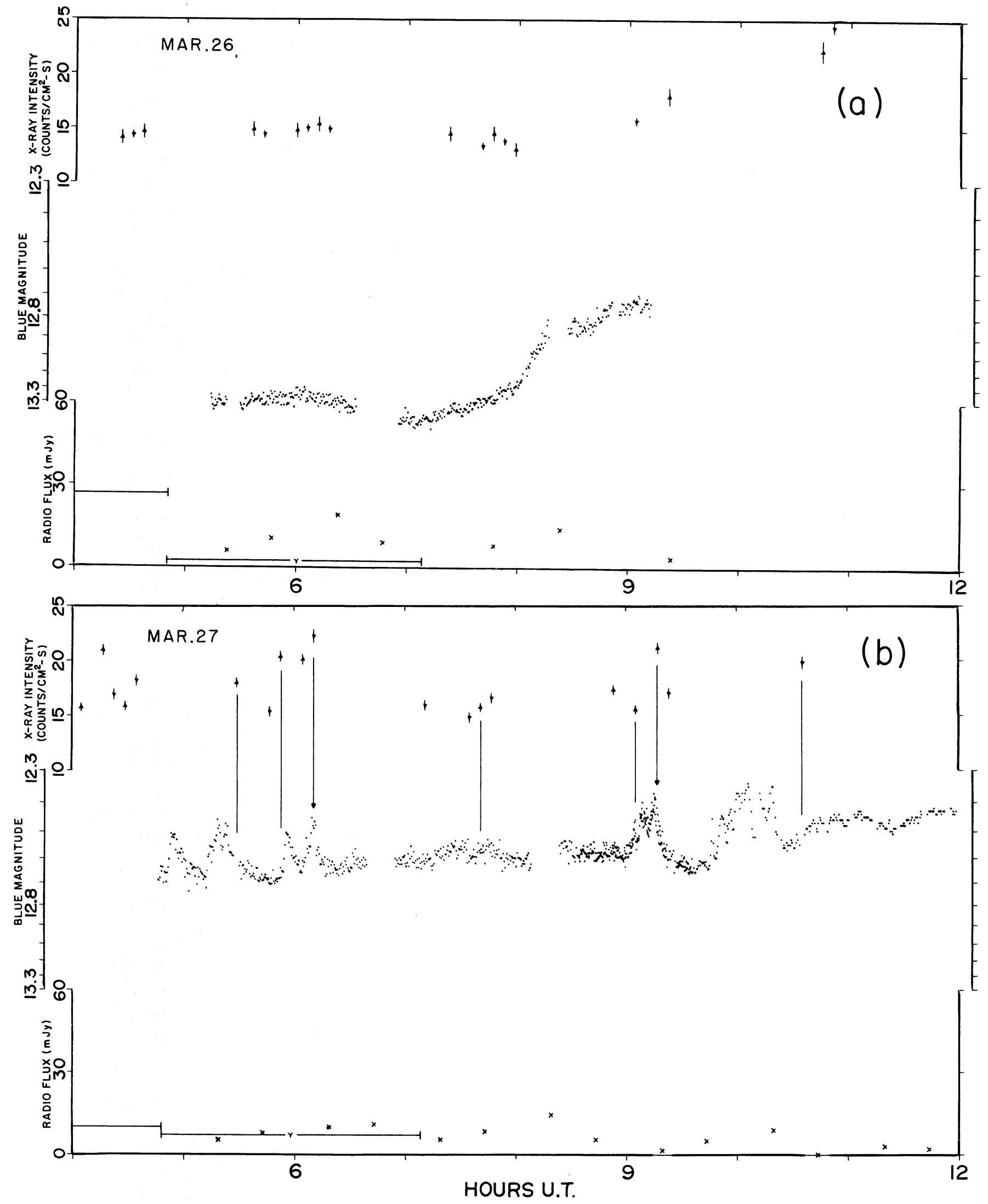

FIG. 7.-Expanded segments of the data, Mar. 26 and Mar. 27. Note the optical change of state followed by an X-ray increase in brightness on Mar. 26. See legend to fig. 5. 

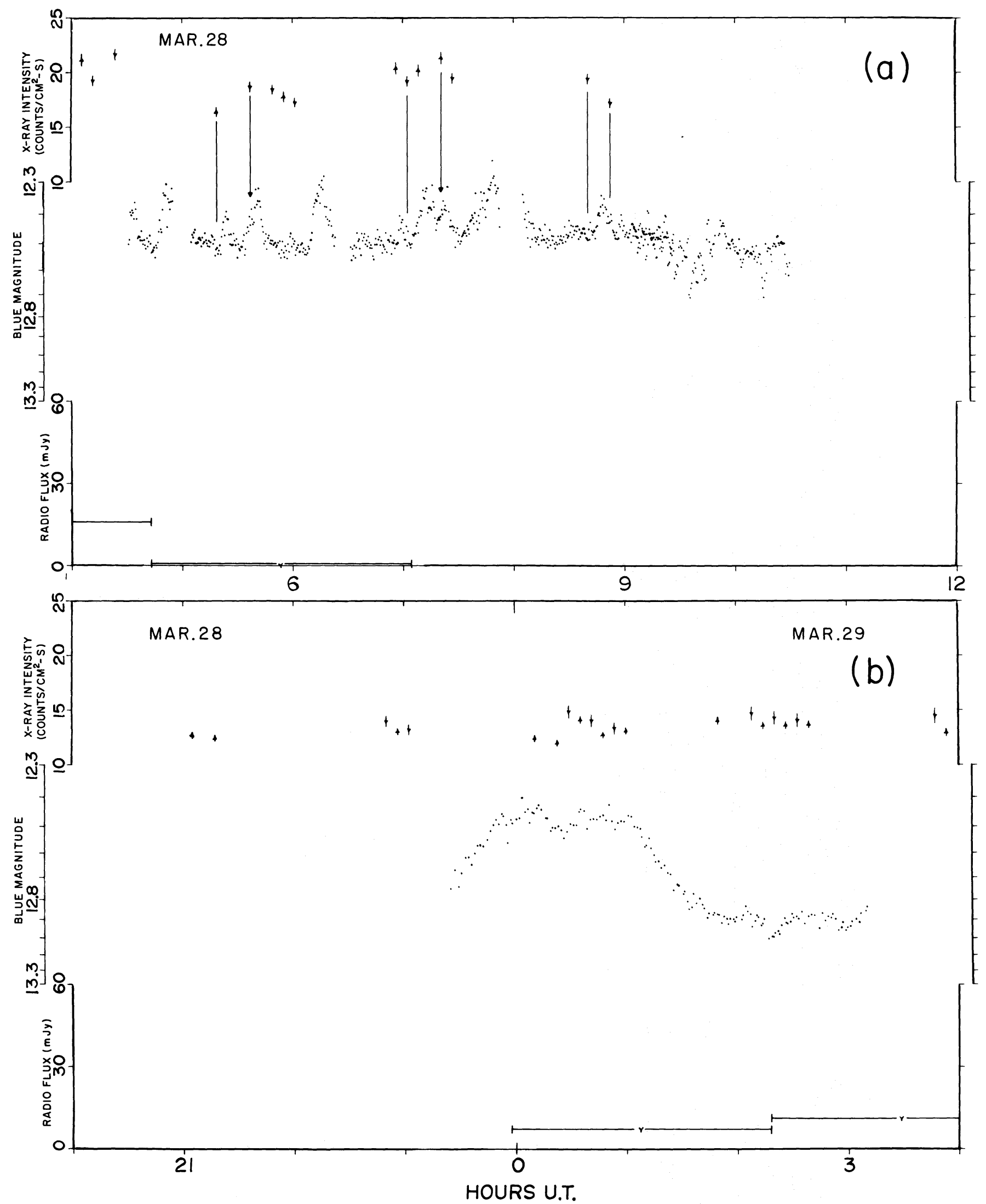

Fig. 8.-Expanded segments of the data, Mar. 28 and Mar. 29. See legend to fig. 5.

454 

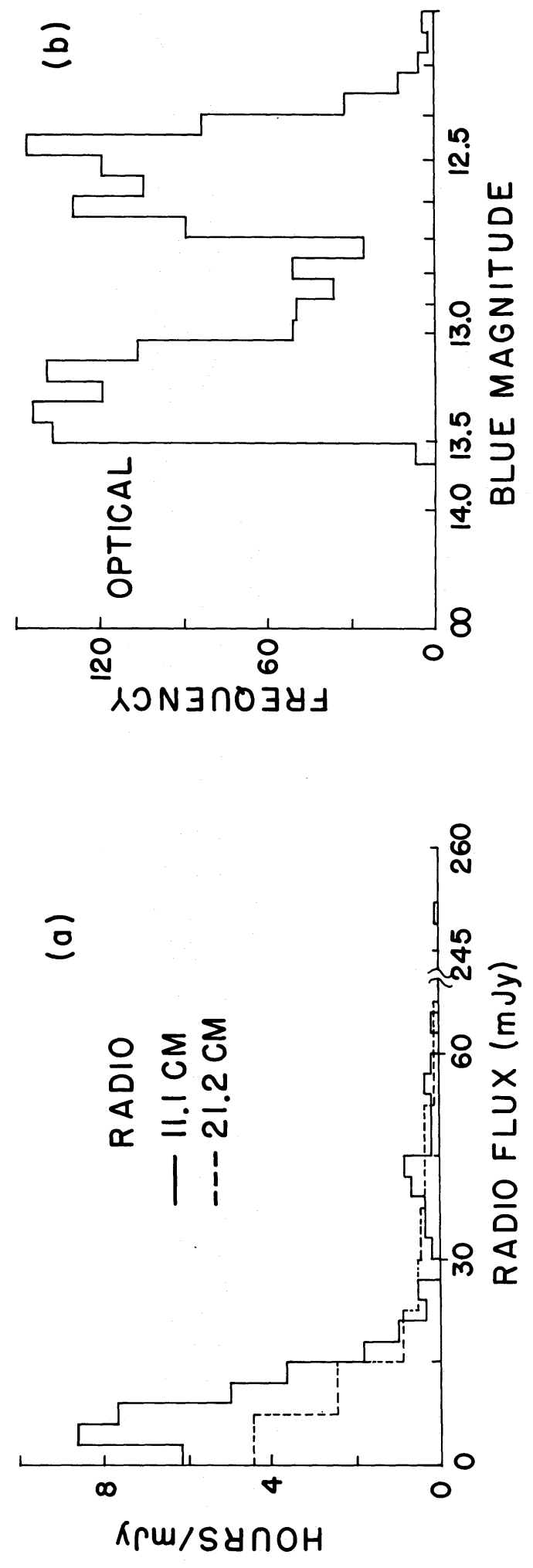

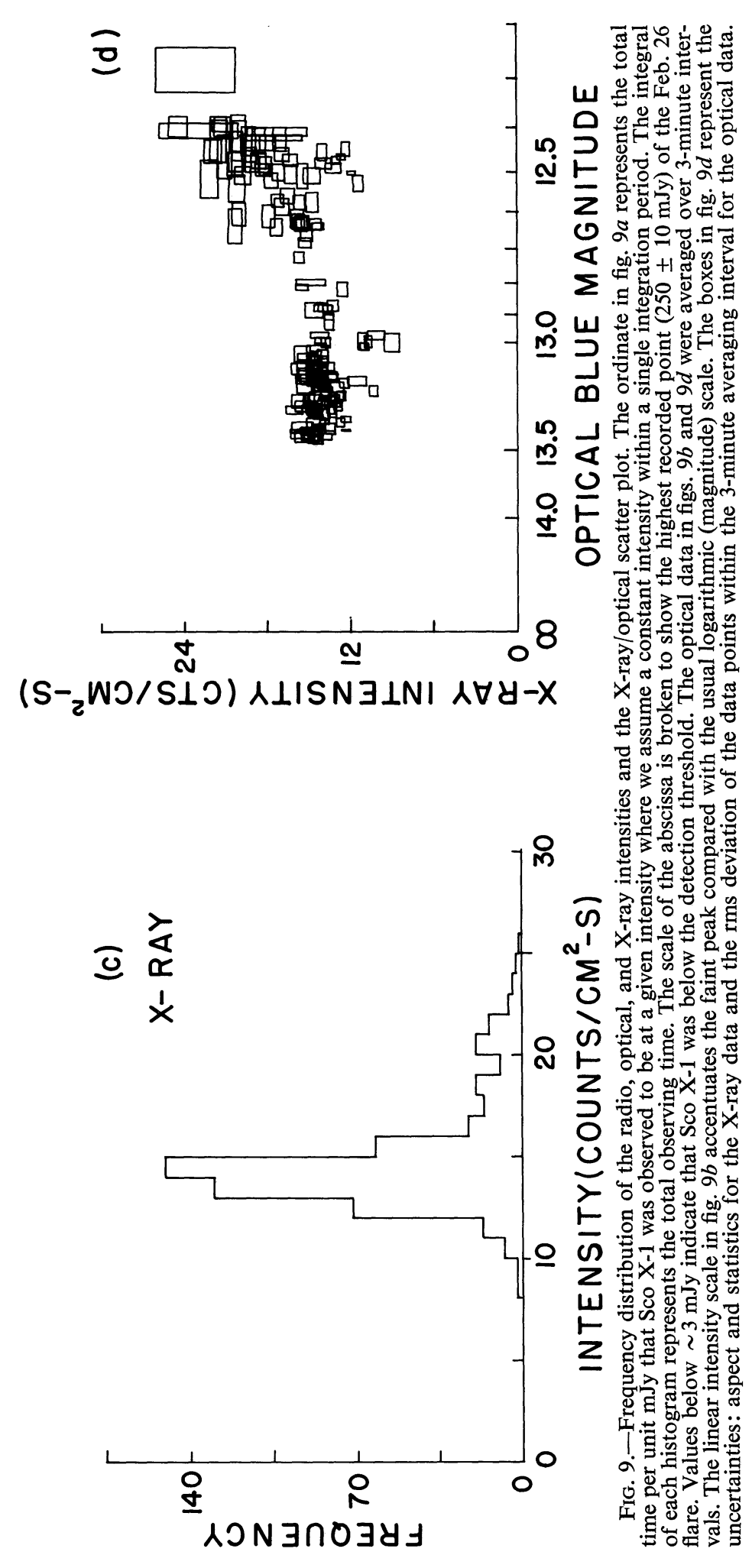

455 
